\title{
ELSEMINARIO Y LA CLASE MAGISTRAL, INFLUENCIA DE LOS MÉTODOS DIDÁCTICOS EN EL RENDIMIENTO ACADÉMICO DE LOS ESTUDIANTES DE PREGRADO
}

\author{
Mario Villegas Yarleque ${ }^{1}$, Priscila Estelita Luján Vera, ${ }^{2}$ Luis Ramón Trelles Pozo ${ }^{3}$, \\ Marlón Martín Mogollón Taboada ${ }^{4}$ \\ mvillegas@unf.edu.pe ${ }^{1}$,priscilavera930@gmail.com², 1trelles@unf.edu.pe ${ }^{3}$, mmogollón@unf.edu.pe \\ ORCID: https://orcid.org/0000-0001-5572-137211, ORCID: https://orcid.org/0000-0002-1359-5715², \\ ORCID: https://orcid.org/0000-0002-5523-203133, ORCID: https://orcid.org/0000-0002-5418-91664 \\ Universidad Nacional de Frontera \\ Sullana-Perú
}

Recibido (03/08/20), Aceptado (14/08/20)

\begin{abstract}
Resumen: El propósito de la investigación fue determinar la influencia de los métodos didácticos de seminario y clase magistral, en el rendimiento académico de los estudiantes de pregrado del curso de Economía General de la Universidad San Pedro de la ciudad de Sullana-Perú. El diseño metodológico es de tipo experimental, de nivel explicativo, de corte longitudinal, utilizando una muestra de 32 estudiantes, repartidos en dos grupos; el primero (G1) de 17 estudiantes de la escuela profesional de Ingeniería Industrial y el segundo (G2) de 15 estudiantes de la escuela profesional de Arquitectura. En los resultados se obtuvo que el promedio de notas después de la aplicación del seminario y la clase magistral es de 12.82 y 11.07 respectivamente. Se concluyó que el rendimiento académico alcanzado por el grupo de estudiantes al que se aplicó el método del seminario es más alto que del grupo de estudio al que se aplicó el método de la clase magistral.
\end{abstract}

Palabras Clave: Seminario, clase magistral, métodos didácticos.

\section{THE SEMINAR AND THE MASTER CLASS, INFLUENCE OF TEACHING METHODS ON THE ACADEMIC PERFORMANCE OF UNDERGRADUATE STUDENTS}

\begin{abstract}
The purpose of the research was to determine the influence of seminar and master class teaching methods on the academic performance of undergraduate students of the General Economics course at San Pedro University. The methodological design is experimental, of an explanatory level, longitudinal, using a sample of 32 students, divided into two groups; the first (G1) of 17 students from the professional school of Industrial Engineering and the second (G2) of 15 students from the professional school of Architecture. In the results it was obtained that the average of marks after the application of the seminar and the master class is 12.82 and 11.07 respectively. It was concluded that the academic performance achieved by the group of students to which the seminar method was applied is higher than that of the study group to which the lecture method was applied.
\end{abstract}

Keywords: Seminar, master class, teaching methods 


\section{I.INTRODUCCIÓN}

La enseñanza universitaria es la manera planificada de la acción educativa en el contexto del nivel superior y tiene como elementos fundamentales; el profesor, el discente y el método de enseñanza-aprendizaje [1]. Se le atribuye a la enseñanza universitaria características que la definen como un proceso constante de indagación [2], obtención y cimentación de conocimientos científicos, así como un procedimiento intelectual que ejerce juicios críticos sobre estos conocimientos nuevos. [3]. Aporta a enriquecer el saber, profundizando en las experiencias vividas y en el empirismo de cada estudiante, y mediante los métodos didácticas del docente universitario establece un formato de enseñanza que direcciona a desarrollar competencias investigativas, que se consolidan en las experiencias del aprendizaje formativo. Estas acciones se denotan en el quehacer del docente universitario, obteniendo como resultado un trabajo coherente y dinámico que se ve reflejado en el proceso de enseñanza [4].

El desarrollo de métodos didácticos en la enseñanza universitaria orienta a que los docentes hagan uso de herramientas pedagógicas variadas, que mejoren su práctica aúlica, más aún en el tiempo de confinamiento en el que nos encontramos por el COVID 19, en donde la virtualización de la educación ha obligado a los docentes peruanos a desarrollar métodos asíncronos y sincrónicos, para poder seguir brindado un proceso de enseñanza de calidad. Frente a lo expuesto el objetivo del estudio es determinar la influencia de los métodos didácticos de seminario y clase magistral, en el rendimiento académico de los estudiantes de pregrado del curso de Economía General de la Universidad San Pedro de la ciudad de Sullana-Perú

La metodología de la presente investigación se centró en el enfoque cuantitativo y el diseño experimental, donde se aplicó una prueba de pretest para medir el rendimiento académico de los estudiantes del curso de Economía general de los programas de estudio de Ingeniería Industrial y Arquitectura de la Universidad San Pedro de la ciudad de Sullana-Perú. Así mismo, se desarrolló un método didáctico distinto para cada grupo, en el G1 conformado por los estudiantes de Ingeniería Industrial se aplicó el método didáctico de seminario, mientras que en el G2 conformado por los estudiantes de Arquitectura se aplicó la clase magistral. El tiempo de aplicación de los métodos didácticos hace referencia a las 16 semanas que dura el semestre académico. Durante este proceso la evaluación académica consistió en la escala vigesimal de $0-20$, estructurada a través de exámenes, lista de cotejo, guías de evaluación e investigación formativa, cuyos procesos se programaron pre- viamente en el silabo del curso.

Cabe indicar que en los resultados se evidencia que el grupo de estudiantes al que se le aplicó el método de seminario obtuvo un rendimiento académico mayor al grupo que se le aplico el método de la clase magistral, ésto debido a que en el seminario los estudiantes tuvieron la oportunidad de ser gestores de sus propios conocimientos, a través de la planificación, organización, sistematización e interacción de las temáticas trabajadas en el curso, llevándolos a internalizar de una forma dinámica los conocimientos adquiridos, mientras que en la clase magistral la monotonía de la clásica estructura de una sesión de clase universitaria no motivo en demasía a los estudiantes.

Arribando a la conclusión que el método del Seminario influye en el rendimiento académico en mayor escala que el método de la clase magistral, evidenciándose en las mejores calificaciones obtenidas por el primero.

\section{II.DESARROLLO}

La universidad es fuente íntegra de conocimiento y por tanto las exigencias que de ella demande, es para desarrollar una educación de calidad, aportando a la evolución de la sociedad por medio de la colaboración de los estudiantes [5]. La metodología y el recurso que utilice una entidad de educación superior para trabajar los saberes, delimitará su establecimiento a través de la historia y su competencia para direccionar y dinamizar el trabajo en aula [6].

La didáctica es una estrategia que la integran métodos variados, para aplicar en el proceso de enseñanza de forma eficaz [7]. Cabe señalar que esta conceptualización no debe reducirse solo a instrumentos específicamente pedagógicos [8], ya que minimizaría la interdisciplinariedad entre los saberes y la intersección social.

El seminario está integrado por grupos de personas que tienen un fin [9], que es la investigación o el intenso estudio en programas organizados, siendo considerado uno de los métodos más importantes de la enseñanza en educación superior. Se especifica que es el desarrollo de una guía orientada de forma previa por el docente, se considera que este tipo de estrategia de aprendizaje es relevante por el sentido de enriquecimiento de los conocimientos adquiridos a través de la investigación que los estudiantes desarrollan [10]; sin olvidar [11] que son los estudiantes, los protagonistas de esta actividad al procesar la información y planificarla para el desarrollo de una exposición, discusión, análisis e intercambio de nociones y juicios críticos.

La técnica didáctica de los seminarios, en su expresión tradicional está vinculada al análisis y discusión del material organizado por los estudiantes y en el caso 
del seminario de problemas su punto de partida es la bibliografía [12], lo que permitirá resolver un problema como inicio de la actividad, es necesario [13] la participación de los estudiantes en el proceso de planificación y organización, que tendrá como resultado el desarrollo de las competencias planteadas en el área de estudio.

La clase magistral es la forma usualmente utilizada en las aulas de una universidad [14], constituye una ocupación protocolar donde el expositor fluye interactivamente con sus estudiantes, exponiendo una mezcla de análisis y caracterización de un tema específico. Tiene como fin último llegar a reflexionar sobre lo expuesto. Cabe indicar que el expositor es un profesional preparado, con dominio escénico y conocimientos de la temática planteada, con capacidad de adaptación de su discurso a un público determinado [15].

El rendimiento académico es una dimensión que indica el logro de las competencias de los estudiantes, que denota todos los saberes adquiridos en el periodo de su formación superior así como sus capacidades para responder a los incentivos del sistema de educación superior [16]. Se vuelve un índice importante al indicar el nivel de logro de los estándares de calidad impuestos por la institución reguladora de la educación superior universitaria [17], es necesario aclarar que referirse a rendimiento académico involucra interpretaciones cuantitativas como cualitativas, que son producto de todo un proceso de enseñanza aprendizaje, donde implica una serie de estrategias y métodos planificados con determinado tiempo, proceso que evidencia el resultado del funcionamiento del sistema educativo de una nación [18].

El rendimiento académico considerado como el avance alcanzado por los estudiantes [19], sin embargo se ve afectado a consecuencia de factores psicológicos innatos del estudiante, producto del sistema social o emocional [20], que puede causarle un círculo de estrés; específicamente en aquellos que no logran superar los infortunios del sistema de evaluación. Ante lo indicado se evidencia que el rendimiento académico es el resultado del gran esfuerzo y sacrificio que hacen los estudiantes en su etapa académica para lograr alcanzar un objetivo, que es culminar la etapa universitaria [21].

\section{III.METODOLOGÍA}

Se desarrolló un proceso de investigación de enfoque cuantitativo, el diseño metodológico es de tipo experimental, de nivel explicativo, de corte longitudi- nal, porque las unidades de estudio son medidas en dos oportunidades (pretest y postest).

El grafico del diseño de la investigación está determinado por la siguiente estructura:

\section{NRG1 O X1 O \\ NRG2 O X2 O}

Figura 1. Estructura del diseño de la investigación

Donde NR significa no randomizado, G1: grupo $\mathrm{n}^{\circ}$ 1 conformado por los estudiantes de Ingeniería Industrial, G2: grupo $\mathrm{n}^{\circ} 2$ conformado por los estudiantes de la clase magistral, O: medición de la observación del pretest y postest, X1: Tratamiento de seminario aplicado al grupo $\mathrm{n}^{\circ} 1, \mathrm{X} 2$ : tratamiento de la clase magistral aplicado al grupo $\mathrm{n}^{\circ} 2$.

La población de estudio estuvo determinada por los estudiantes de la escuela profesional de Ingeniería Industrial y la escuela profesional de Arquitectura. La muestra es no probabilística, conformada por 32 estudiantes del curso de Economía General de la Universidad San Pedro de la ciudad de Sullana-Perú, repartidos en dos grupos: grupo uno (G1) que pertenece a la escuela profesional de Ingeniería Industrial y que son en total 17 unidades de estudio y grupo dos (G2) que pertenece a la escuela profesional de Arquitectura, siendo en total 15 unidades de estudio.

Para la recolección de información se utilizó como técnica la prueba y/o encuesta de conocimiento, pretest y postest, en cual contendrá cuestionamientos referidos a los temas aplicados durante el tratamiento. Así mismo, tabla de observación y cotejo, para evaluar las variables; en ella, se hará evidencia de las acciones realizadas por los estudiantes, así como también las acciones desarrolladas por el docente, durante la aplicación del tratamiento.

\section{IV.RESULTADOS}

Realizado el análisis estadístico, después de la aplicación de la prueba de pretest para el G1 conformado por los 17 estudiantes de Ingeniería Industrial se obtuvo que las calificaciones O1(calificación de pretest) oscilan entre 2 y 9 puntos en la escala vigesimal, lo que indica un rendimiento bajo, donde fi: frecuencia absoluta, FI: frecuencia absoluta acumulada, hi: frecuencia relativa, $\mathrm{HI}$ frecuencia relativa acumulada y $\%$ hi: frecuencia relativa porcentual (esta nomenclatura aplica para las Tablas I, II, IV y V) 
Tabla I. Pretest del grupo $\mathrm{n}^{\circ} \mathbf{1}$

\begin{tabular}{cccccc}
\hline $\begin{array}{c}\text { Calificación de } \\
\text { Pretest (O1) }\end{array}$ & fi & Fi & hi & Hi & \% hi \\
\hline $\mathbf{2}$ & 2 & 2 & 0,12 & 0,12 & 12 \\
$\mathbf{3}$ & 3 & 5 & 0,18 & 0,30 & 18 \\
$\mathbf{4}$ & 2 & 7 & 0,12 & 0,42 & 12 \\
$\mathbf{6}$ & 2 & 9 & 0,12 & 0,54 & 12 \\
$\mathbf{7}$ & 1 & 10 & 0,06 & 0,60 & 6 \\
$\mathbf{8}$ & 6 & 16 & 0,34 & 0,94 & 34 \\
$\mathbf{9}$ & 1 & 17 & 0,06 & 1,00 & 6 \\
TOTAL & 17 & & 1,00 & & 100 \\
\hline
\end{tabular}

Fuente: Analisis de pretest.

En la tabla II se puede observar los resultados obtenidos después de la aplicación de la prueba de pretest para el G2 conformado por 15 estudiantes de Arquitectura, donde se evidencia que las calificaciones del rendimiento académico oscilan entre 2 y 8 puntos.

Tabla II. Pretest del grupo $\mathrm{n}^{\circ} \mathbf{2}$

\begin{tabular}{cccccc}
\hline $\begin{array}{c}\text { Calificación de } \\
\text { Pretest (O1) }\end{array}$ & fi & Fi & hi & Hi & \% hi \\
\hline $\mathbf{2}$ & 3 & 3 & 0,20 & 0,20 & 20 \\
$\mathbf{3}$ & 1 & 2 & 0,07 & 0,27 & 7 \\
$\mathbf{4}$ & 2 & 6 & 0,13 & 0,40 & 13 \\
$\mathbf{5}$ & 2 & 8 & 0,13 & 0,53 & 13 \\
$\mathbf{6}$ & 4 & 12 & 0,27 & 0,80 & 27 \\
$\mathbf{7}$ & 1 & 13 & 0,07 & 0,87 & 7 \\
$\mathbf{8}$ & 2 & 15 & 0,13 & 1,00 & 13 \\
TOTAL & 15 & & 1,00 & & 100 \\
\hline
\end{tabular}

Fuente: Análisis de pretest.

En la tabla III, se evidencia que en el pretest de ambos grupos la media de las calificaciones de un total de 17 estudiantes del grupo $\mathrm{n}^{\circ} 1$ obtuvieron 5,70 y la media de las calificaciones de un total de 15 estudiantes del grupo $\mathrm{n}^{\circ} 2$ obtuvieron 4,93. En los dos casos es una nota desaprobatoria, lo cual nos indica que los estudiantes de los dos grupos tienen niveles de conocimientos previos muy similares. En el caso del coeficiente de variación del grupo 1 es de $43.68 \%$, considerado como muy alto y del grupo 2 es de 41,58 \%, también considerado como alto, indicando que las notas de los grupos, antes de la aplicación de seminario y la clase magistral, son muy dispersas entre sí, es decir las notas o rendimiento académico es heterogéneo. Lo que es corroborado con la prueba de hipótesis de pretest que nos arroja una T calculada de 0.942 y una T tabulada de 2.042 que nos lleva a aceptar la hipótesis que no existen diferencias significativas entre los dos grupos de estudio antes de la aplicación del método didáctico de seminario.

Tabla III. Resumen estadígrafos del pretest

\begin{tabular}{ccc}
\hline Grupo estadígrafo & G1 & G2 \\
\hline Media & 5,70 & 4,93 \\
Desviación estándar & 2,49 & 2,05 \\
Coeficiente de variación & $43,68 \%$ & $41,58 \%$ \\
Total de alumnos & 17 & 15 \\
\hline
\end{tabular}

Fuente: Elaboración, análisis e interpretación del pretest.

En la tabla IV se denota el rendimiento académico del grupo $\mathrm{n}^{\circ} 1$ de los estudiantes de Ingeniería Industrial después de la aplicación del método didáctico de seminario, donde se medió y evaluó el nivel de conocimientos que adquirieron durante la aplicación del tratamiento, esta medición se realizó a través de la prueba de postest, obteniendo como resultados calificaciones entre 10 y 15 puntos.

Tabla IV. Postest del grupo $\mathrm{n}^{\circ} 1$

\begin{tabular}{lccccc}
\hline $\begin{array}{c}\text { Calificación de } \\
\text { Postest (O2) }\end{array}$ & $\mathbf{f i}$ & $\mathbf{F i}$ & $\mathbf{h i}$ & $\mathbf{H i}$ & $\mathbf{\%} \mathbf{h i}$ \\
\hline $\mathbf{1 0}$ & 2 & 2 & 0,12 & 0,12 & 12 \\
$\mathbf{1 1}$ & 2 & 4 & 0,12 & 0,24 & 12 \\
$\mathbf{1 2}$ & 3 & 7 & 0,18 & 0,42 & 18 \\
$\mathbf{1 3}$ & 3 & 10 & 0,18 & 0,60 & 18 \\
$\mathbf{1 4}$ & 4 & 14 & 0,22 & 0,82 & 22 \\
$\mathbf{1 5}$ & 3 & 17 & 0,18 & 1,00 & 18 \\
TOTAL & 17 & & 1,00 & & 100 \\
\hline
\end{tabular}

Fuente: Análisis de postest.

En la tabla $\mathrm{V}$ se evidencia el rendimiento académico del grupo $\mathrm{n}^{\circ} 2$ de los estudiantes de Arquitectura después de la aplicación del método didáctico de la clase magistral, donde se medió y evaluó el nivel de conocimientos 
Tabla V. Postest del grupo $\mathrm{n}^{\circ} 2$

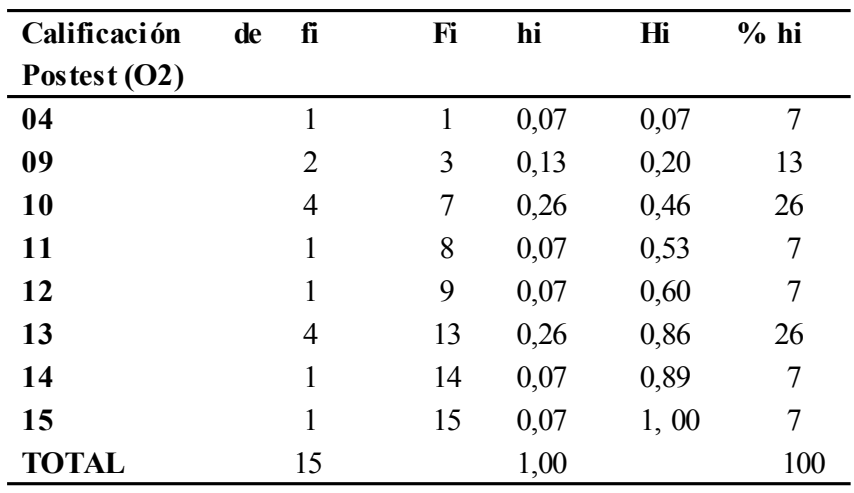

Fuente: Análisis de postest.

En la tabla IV, se evidencia que en el postest de ambos grupos la media de las calificaciones de un total de 17 estudiantes del grupo $\mathrm{n}^{\circ} 1$ obtuvieron 12.83 en la escala vigesimal y la media de las calificaciones de un total de 15 estudiantes del grupo $n^{\circ} 2$ fue 11,07 en la escala vigesimal, observándose que son notas aprobatorias y que las notas del grupo 1 son más altas que las del grupo 2, ello debido a la aplicación del método del seminario.

La prueba de hipótesis para la diferencia de medias del postest, arroja una $\mathrm{T}$ calculada $\mathrm{Tc}=2.337 \mathrm{y}$ una $\mathrm{T}$ tabulada $\mathrm{Tt}=2.042$, esto nos lleva a aceptar la hipótesis de que existen diferencias significativas entre las medias de los grupos de los estudiantes de Ingeniería Industrial y de Arquitectura, los cuales difieren significativamente después de la aplicación del método de seminario y del método de la clase magistral, respectivamente.

Tabla VI. Resumen estadígrafos del postest

\begin{tabular}{lll}
\hline $\begin{array}{l}\text { Postest } \\
\text { Estadígrafo }\end{array}$ & G1 & G2 \\
\hline Media aritmética & 12.23 & 11.07 \\
Desviación estándar & 1.71 & 2.71 \\
Varianza & 2.94 & 7.34 \\
Total de alumnos & 15 & 17 \\
\hline
\end{tabular}

Fuente: Elaboración, análisis e interpretación de postest.

\section{V.CONCLUSIÓN}

El método didáctico de seminario influye significativamente en el rendimiento académico de los estudiantes del curso de Economía General de la Universidad San
Pedro de la ciudad de Sullana Perú, cuyos resultados se evidencian en las calificaciones obtenidas durante el proceso de aplicación. Esto debido a la forma interactiva que experimentan los estudiantes en la organización y planificación de los conocimientos en cada seminario. A diferencia de la clase magistral en donde las calificaciones evidencian un bajo rendimiento académico, demostrando su obsolescencia, ya que el estudiante es un receptor pasivo de información. Cabe resaltar que el seminario es un método aplicable a todas las materias, ya que su forma expositiva permite la comprensión de la temática y al mismo tiempo la participacion activa y retroalimentativa de los estudiantes, generando en ellos una forma más sencilla de aprender. De tal manera que para mejorar la práctica pedagógica aúlica en el sistema universitario se debe implementar métodos didácticos que complementen y diversifiquen los procesos de enseñanza-aprendizaje como el seminario, aprendizado basado en problemas, aprendizaje colaborativo y aprendizaje basado en proyectos, de tal manera que induzcan a los estudiantes por los caminos de la investigación.

\section{REFERENCIAS}

[1]J. L. Llanos Castilla, «La enseñanza universitaria, los recursos didácticos y el rendimiento académico de los estudiantes de la E.A.P. Educación de la UNMSM,» Maestría Thesis, Universidad Nacional Mayor de San Marcos, Lima, Perú, 2012.

[2]A. Torres Soto y M. Vallejo Ruiz, «¿Contribuye la universidad al desarrollo de los aprendizajes de calidad? Estudio descriptivo con estudiantes de la Universidad de Murcia,» Revista Electrónica Interuniversitaria de Formación del Profesorado, vol. 21, no 1, pp. 129142, 2018.

[3]E. Barrientos Jiménez, «Didáctica de la Educación Superior,» compilación, UPG. U.N.M.S.M. Facultad de Educación, pp. 144-145, Lima, 2008.

[4]C. Figueroa y G. N. Sanjinés Tudela, «Una mirada a la didáctica de la universidad y las ciencias sociales. Análisis comparativo,» Revista Espacios, vol. 39, nº 46, pp. 36, 2018.

[5]H. Damaris Díaz, «La Didáctica Universitaria: Referencia imprescindible para una enseñanza de calidad,» Revista Electrónica Interuniversitaria de formación del profesorado, vol. 2, nº 1, pp. 107-116, 1999.

[6]P. A. Duque, S. L. Vallejo A. y J. C. Rodríguez L. , «Prácticas Pedagógicas y su relación con el desempeño pedagógico,» Maestría Thesis, Universidad de Manizales, Manizales, Colombia, 2013.

[7]B. Y. Hernández Sánchez, I. D. Toro Jaramillo y E. Alarcón Meza, «La Educación y la Didáctiva en el contexto del saber administrativo,» Revista Espacios, vol. 
37, nº 17, pp. E4, 2016.

[8]M. A. Villalta Paucar y S. Martinic Valencia, «Modelos de estudio de la interacción didáctica en la sala de clase,» Investigación y Posgrado, vol. 24, no 2, pp. 61-76, 2009.

[9]C. N. Piña Loyola, A., Seife Echevarría y C. M. Rodríguez Borrell, «El seminario como forma de organización de la enseñanza,» MediSur, vol. 10, $\mathrm{n}^{\mathrm{o}} 2$, pp. 109-116, 2012.

[10]M. d. Céu Taveira y M. De Nazaré Loureiro, «Calidda de la Educación Superior y Orientación: Eficacia de un Seminario de Gestión Personal de la Carrera para Pre Graduados,» Revista Eelctrónica Interuniversitaria de Formación del Profesorado, vol. 17, nº 3, pp. 93-104, 2014.

[11]M. Gómez Salinas , «Factores socioeconómicos y pedagógicos que inciden en el rendimiento académico en estudiantes de la Carrera de Ciencias Sociales, UNAN-CUR Matagalpa, durante el I semestre 2008,» Maestría Thesis, Universidad Nacional Autónoma de Nicaragua, Managua, Nicaragua, 2010.

[12]J. Arias Trujillo y R. Porras Soriano , «Los seminarios de problemas como estrategia docente en las enseñanzas técnicas: Una experiencia aplicada a la Ingeniería del Terreno,» Revista de Docencia Universitaria , vol. 11, pp. 47-64, 2013.

[13]R. Cano Gonzales, «Tutoría universitaria y aprendizaje por competencias ¿Como lograrlo?,» Revista Electrónica Interuniversitaria de formación del profesorado, vol. 12, $\mathrm{n}^{\mathrm{o}} 1$, pp. 181-204, 2009.

[14]M. Jiménez Ruíz, H. Rodríguez Navarro, S. Sánchez Fuentes y J. Rodríguez Medina , «Construcción del discurso en torno a la Educación Inclusiva,» Revista Electrónica Interuniversitaria de Formación del Profesorado, vol. 21, $\mathrm{n}^{\mathrm{o}}$ 1, pp. 185-217, 2018.

[15]M. F. Elgueta Rosas y E. E. Palma Gonzáles, «Una propuesta de clasificación de la clase magistral impartida en la facultad de Derecho,» Revista Chilena de Derecho, vol. 41, no 3, pp. 907-924, 2014.

[16]K. P. Pérez, «Influencia de la función ejecutiva en el rendimiento académico de estudiantes universitarios. Caso Fundación Universitaria Tecnológico Comfenalco, » Revista Espacios, vol. 40, no 8, p. 7, 2019.

[17]P. E. Luján Vera, L. R. Trelles Pozo y M. M. Mogollón Taboada, «Asertividad y rendimiento académico en estudiantes de la facultad de ciencias administrativas de la Universidad Nacional de Piura,» UCV-SCIENTIA , vol. 11, n ${ }^{\mathrm{o}}$ 1, pp. 13-20, 2020.

[18]G. Paredes , «B-Learning y su influencia en el rendimiento académico en los esttudiantes de la asignatura de Seminario de tesis de la facultad de Ingeniería de Sistemas e Informática de la Universidad Nacional de
San Martín-Tarapoto,» Tesis, Universidad Nacional de San Martín, Tarapoto, Perú, 2012.

[19]E. V. Tapia Sosa (2019). La incidencia de las insuficiencias de lecto-escritura en el aprendizaje y rendimiento escolar. Revista Universidad, Ciencia y Tecnología, vol. 2 (2), pp. 75-83.

[20]M. P. Redondo y L. K. Jimenéz, «Autoconcepto y rendimiento académico en estudiantes de secundaria en la ciudad de Valledupar-Colombia,» Revista Espacios, vol. 41, no 9, pp. 17, 2020.

[21]P. S. Obando Castro, «Aprendizaje colaborativo en el rendimiento Académico y Habilidades Sociales en el Programa de Especialización en Enfermería,» Rev. Enfermería Herediana, vol. 2, no 1, pp. 32, 2009.

\section{RESUMEN CURRICULAR}

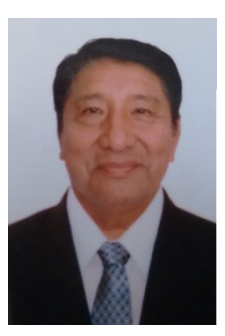

Mario Villegas Yarleque, Economista con maestría en Ciencias de la Educación Superior, con experiencia en el sector privado en empresas de transporte de pasajeros y sector público, en gobiernos locales y regionales; con 14 años de experiencia académica en universidades públicas y privadas. Actualmente me desempeño como docente auxiliar a tiempo completo en la Universidad Nacional de Frontera en la Facultad de Ingeniería Económica desde Marzo del 2017 hasta la actualidad, en calidad de docente nombrado, E-mail: mvillegas@unf.edu.pe

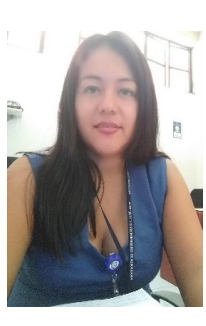

Priscila Estelita Luján Vera, Licenciada en Educación Especialidad Lengua y Literatura. Magister en Docencia Universitaria. Doctorada en Ciencias de la Educación. Experiencia profesional enfocada en la planificación y ejecución de proyectos de Innovación en Ciencias Sociales, en proyectos de gestión Social y Turísticos. Profesional proactica, dinámica, con capacidad de Liderazgo. E-mail: priscilavera930@gmail.com

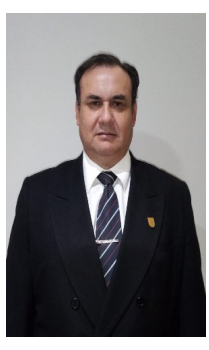

Luis Ramón Trelles Pozo, Economista, con maestría en administración, Mención General Empresarial. Mi formación profesional se ha orientado hacia el conocimiento de la Realidad Socio- Económica y Educativa de la Región Piura, participando activamente en diversas instituciones en la elaboración y evaluación de proyectos, comercialización, abastecimiento, encuestas, programación y estadística. Docente nombrado de la Universidad Nacional de Frontera.E-mail: ltrelles@unf.edu.pe

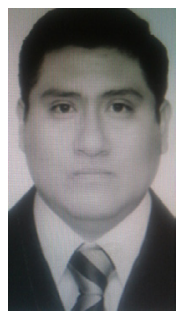

Marlón Martín Mogollón Taboada, Contador Público Colegiado, magister en Investigación y Docencia en la Universidad Nacional Pedro Ruíz Gallo de Lambayeque. Actualmente cursa estudios del tercer ciclo del Doctorado con mención en "Contabilidad y Finanzas" en la Universidad Nacional de Piura. Asesor independiente en empresas del sector privado en materia contable, tributaria, laboral y financiera. Investigador DINA del Consejo Nacional de Ciencia y Tecnología - CONCYTECE-mail: mmogollón@unf.edu.pe 